\title{
BMJ Open An Exploration of the help-seeking experiences of patients in an allied professions-led rapid access chest pain pathway: a qualitative study
}

Nolan Stain (D) , ${ }^{1}$ Anna Cheshire, ${ }^{2}$ Catherine Ross, ${ }^{3}$ Damien Ridge ${ }^{2}$

To cite: Stain N, Cheshire A, Ross C, et al. An Exploration of the help-seeking experiences of patients in an allied professions-led rapid access chest pain pathway: a qualitative study. BMJ Open 2020;10:e040468. doi:10.1136/ bmjopen-2020-040468

- Prepublication history and additional material for this paper is available online. To view these files, please visit the journal online (http://dx.doi.org/10. 1136/bmjopen-2020-040468)

Received 14 May 2020 Revised 22 0ctober 2020 Accepted 28 October 2020

\section{Check for updates}

(c) Author(s) (or their employer(s)) 2020. Re-use permitted under CC BY-NC. No commercial re-use. See rights and permissions. Published by BMJ.

${ }^{1}$ Cardiac Physiology, Barts Health NHS Trust, London, UK ${ }^{2}$ College of Liberal Arts and Sciences, University of Westminster, London, UK ${ }^{3}$ The Office of Chief Scientific Officer, NHS England, London, UK

Correspondence to

Dr Nolan Stain;

nolanst@aol.com

\section{ABSTRACT}

Objective A number of studies have explored delayed help-seeking practices for acute coronary syndrome (ACS) and have indentified multiple intersecting factors which may play a role, for example, attributing symptoms, age, gender, ethnicity and contextual influences. However, the pathway to diagnosis for suspected coronary heart disease (CHD) symptoms in a rapid access chest pain clinic (RACPC) context is underexplored. The objective of this study was to examine patients' help-seeking experiences of accessing RACPC services, from the point at which they notice and interpret symptoms, to their decision to seek help from their general practitioner.

Design Qualitative study.

Setting Interviews were conducted in the RACPC at Queen Mary's Roehampton Hospital, London, UK. Participants Maximum variation sampling was used to recruit 30 participants ( 15 men and 15 women) referred to a RACPC, using sampling dimensions of age, ethnicity and occupation.

Methods Semi-structured interviews focussed on the patient experience of their pathway to the RACPC. Thematic analysis was used to analyse the interview data. Results Participant interpretation of symptoms was shaped by multiple influences; reluctance to seek help contributed to delay; various factors acted as drivers as well as barriers to help-seeking; and GP referrals to RACPC were based on symptoms as well as patients' need for reassurance.

Conclusion We found complex issues shaped the patient's decision-making when accessing the RACPC, including making sense of symptoms and help-seeking practices. These findings can be used to develop health promotion literature to encourage early help-seeking and improve RACPC services.

\section{BACKGROUND}

Coronary heart disease (CHD) remains a leading cause of mortality, accounting for $42 \%$ of all European deaths. ${ }^{1}$ Rapid clinical evaluation and intervention are known to be critical in improving patient outcomes. ${ }^{2}$ Chest pain is the most prevalent presentation of CHD in both men and women. ${ }^{2-4}$ Importantly, chest pain can have many non-cardiac causes; in $15 \%$ of cases, no origin can be

\section{Strengths and limitations of this study}

- The study explores the pathway into rapid access chest pain clinic (RACPC) among a previously neglected cohort of patients.

- Qualitative methods uncovered in-depth insights into patients' interpretation of symptoms and helpseeking practices.

- Findings can be used to inform RACPC pathway accessibility and service improvements.

- While maximum variation sampling recruited participants with a broad set of demographic characteristics, this single centre study may have under-represented the opinion of individuals with Black ethnicity.

attributed. ${ }^{5}$ As a result, understanding the significance of chest pain and establishing its causality can be complex for patients and health professionals.

In the UK, rapid access chest pain clinics (RACPC) introduced by the National Service Framework for CHD (NSF-CHD), are often used as the initial referral pathway to evaluate the potential causality of chest pain. ${ }^{6}$ Referrals to RACPC originate in primary care via general practitioners (GPs), following a clinical evaluation. RACPC pathways are operated by cardiac scientists and nurses, who undertake advanced practice roles. ${ }^{78}$ In the years following their implementation (2002 to 2006), referral rates to RACPC doubled and deaths from acute CHD events began to decline, ${ }^{9}$ suggesting that the NSF-CHD (2000) programme was effective in improving access to services and enhancing public awareness. However, the ability of the RACPC to reach all potential patients with CHD symptoms may be reduced by suboptimal help-seeking practices.

Help-seeking is a concept used to understand the enablers and barriers to accessing help for health conditions. ${ }^{10}$ Help-seeking 
can be informal through speaking with friends and family, or formal through a consultation with a medical professional. ${ }^{11}$ Informal help-seeking can promote obtaining medical help(e.g. when people are encouraged to speak to a professional ${ }^{1213}$ or delay it when loved ones downplay symptoms for reassurance. ${ }^{14}$ The extent to which informal help-seeking for CHD is sought is influenced by multiple factors, including a reluctance to speak to family members about health symptoms so as not to worry them. ${ }^{12} 14$ The context may also be influential e.g. those present at the time of chest pain onset are more likely to be consulted. ${ }^{15}$

The drivers for medical help-seeking in patients with potential CHD are complex. Several studies have found that the severity of symptoms, perceived significance of symptoms, coronary candidacy (lay perception about who is at risk of CHD) and the ability to access medical services all play a role. Severe symptoms often encourage help-seeking, whereas there is a reluctance to do so for milder symptoms which may be dismissed or initially selfmanaged. ${ }^{16-18}$ The significance attached to symptoms is influenced by one's knowledge of CHD, previous experience of CHD, family history of CHD and worries about one's lifestyle choices. ${ }^{12} 141920$ Popular media and public health campaigns can also influence the interpretation of CHD symptoms and may promote help-seeking by creating awareness. $^{21}{ }^{22}$ However, they can also act as barriers, since patients are reluctant to seek help if symptoms do not align with what is expected. ${ }^{1618}{ }^{23}$ More recently, the COVID-19 pandemic has illustrated how a well-intended media campaign 'Stay home, save lives' ${ }^{\text {'24 }}$ can have unintended impacts on help-seeking for CHD. ${ }^{25}{ }^{26}$ Other barriers to help-seeking include concerns about wasting the doctor's or one's own time with unimportant symptoms ${ }^{1421}$ and difficulties accessing medical services. ${ }^{1419} 27$

Many studies have explored the role of gender in helpseeking for CHD in acute coronary syndrome (ACS). Gender appears to interplay with multiple factors that influence help-seeking including knowledge, personal beliefs, age, ethnicity and socioeconomics. In some studies women are reported to be more likely to delay seeking help than men. ${ }^{22} 28-31$ However, other studies suggest that help-seeking delays occur irrespective of gender and that factors including the severity of symptoms, age, socioeconomics, culture, ethnicity and context may override gender. ${ }^{15} 1718$ Despite this debate around gender, the underdiagnosis and treatment of CHD in women remains a recognised problem. ${ }^{42}{ }^{33}$ A recent European study ${ }^{4}$ highlighted the negative effects on clinical outcomes in women with ACS as a result of delayed help-seeking.

While the ACS patient pathway to diagnosis and treatment is widely reported in the literature ${ }^{2134-36}$ the RACPC context (noticing symptoms, accessing a GP referral and receiving a RACPC diagnosis) remains largely unexamined. Insights into patient pathways to diagnosis for cancer are particularly useful to examine here, as they are potentially reflective of the non-emergency situation for patients attending RACPC. ${ }^{36-39}$ For example, Scott $e t$ al describe 'detection of bodily changes' and 'perceives reason to discuss symptoms with healthcare professionals' as key events in the initial stages. Similar understanding of patients' decision-making and help-seeking experiences in the RACPC pathway could lead to improvements in health education and RACPC services that promote early diagnosis and treatment.

\section{Study aim}

The aim of this study was to explore patients' help-seeking experiences of accessing RACPC services from the point at which they notice and interpret symptoms, to their decision to seek help from their GP and obtain a referral to RACPC.

\section{METHOD AND STUDY DESIGN}

The study adopted an interpretative qualitative approach using semi-structured interviews. ${ }^{40}$ Semi-structured interviews were selected as the best means to capture the participants' interpretation of symptoms, decisionmaking processes and their experiences of GP referral pathways to RACPC. Interviews were conducted face-toface, which is appropriate when dealing with potentially sensitive health conditions . ${ }^{4142}$

The original research protocol for this study was published as 'Gender comparisons in non-acute cardiac symptom recognition and subsequent help-seeking decisions: a mixed-methods study protocol' and included the construction of a help-seeking questionnaire. ${ }^{43}$ As the study evolved the authors decided an in-depth focus on the qualitative approach would provide a better contribution to the research literature. Thus, the latter part of the study described in the original protocol was dropped and the research question was also changed to reflect this. The study is reported here using the Standards for Reporting Qualitative Research (SRQR) guidelines. ${ }^{44}$

\section{Sampling}

The study used maximum variation (MV) sampling to recruit a diverse range of participants from a Londonbased RACPC, subject to inclusion/exclusion criteria (table 1). ${ }^{42}{ }^{45}$ The MV characteristics used in this study were age, sex, ethnicity and occupation. Participants were interviewed (sampled) either prior to receiving the RACPC diagnosis or immediately afterwards. The rationale was to record the varying patient perspectives. A sample of 30 patients (15 men and 15 women) with the desired sampling characteristics were recruited, as detailed in table 2.

\section{Recruitment}

The sample was identified from multiple sources including GP referral letters, medical records and the patient administration system. As MV characteristics were rarely contained in a single source, multiple records were examined to determine suitability. A total of 42 patients were identified. All potential participants were contacted 
Table 1 Inclusion and exclusion criteria

\section{Inclusion criteria}

Males and females over the age of 18 years.

Patients referred to rapid access chest pain clinic (RACPC) for assessment of chest pain (including associated symptoms, that is, arm pain, throat pain and breathlessness).

Only patients able to communicate sufficiently in English .

Able and willing to consent to the study.

\section{Exclusion criteria}

Patients presenting with an acute cardiac event.

Patients not accessing the RACPC. For example, patients referred directly to cardiac outpatients or treated as a acute coronary syndrome (ACS).

Patients unable to give consent for whatever reason or are deemed unable to give consent, as it would be unethical to include them.

by the researcher or senior member of the clinical team by telephone in advance of their RACPC appointment, and were invited to participate in an interview. Three patients chose not to participate at the outset. Participant information sheets and consent forms were sent to 39 patients, all of whom agreed to participate; however, nine of these patients did not complete the interview on the day. All participants provided written consent.

The recruitment process continued until data saturation and the MV sampling criteria were achieved.

Table 2 Recruited sample characteristics

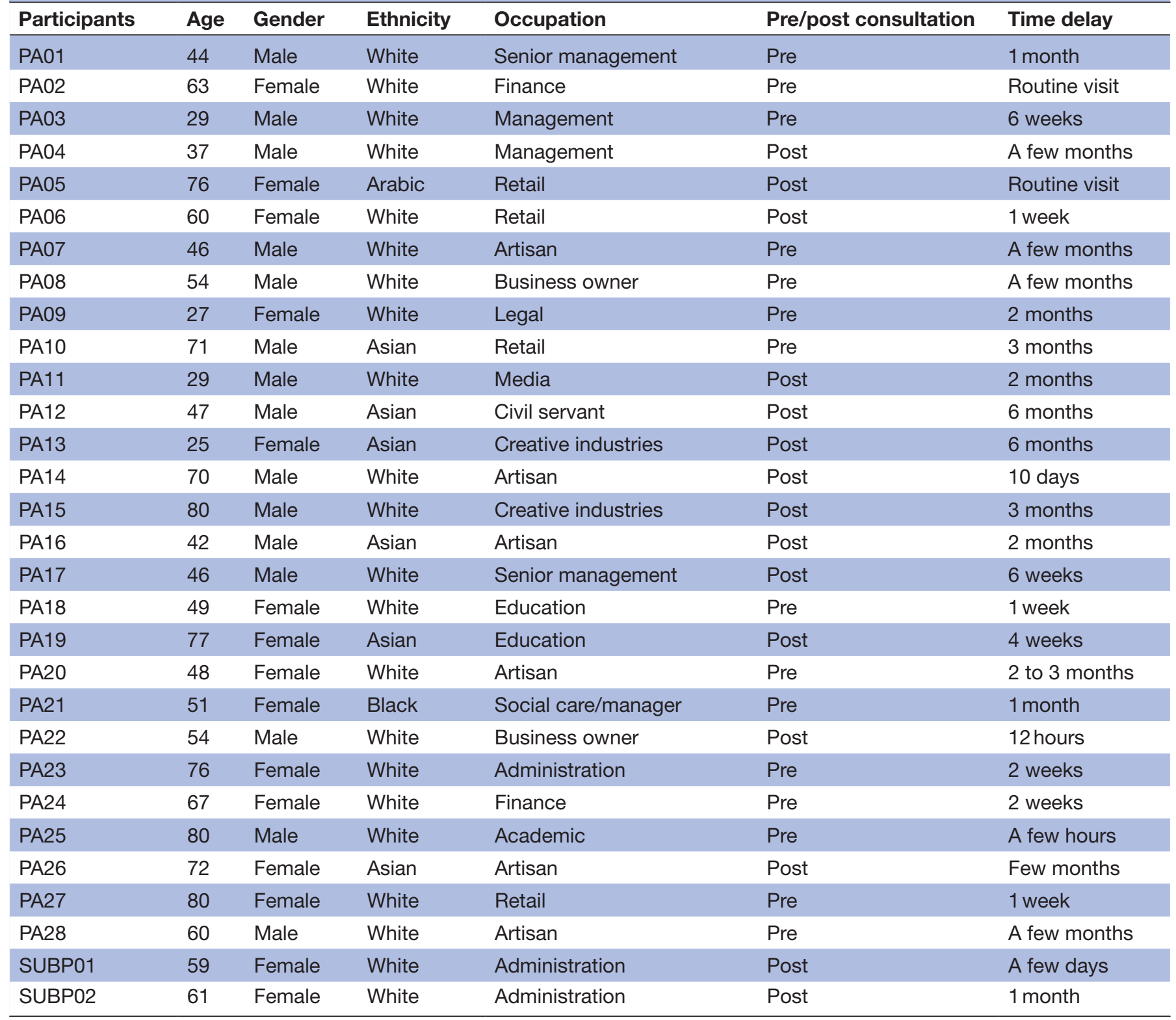


Table 3 Authors characteristics

\begin{tabular}{lllll}
\hline & Nolan Stain (NS) & Anna Cheshire (AC) & Catherine Ross (CR) & Damien Ridge (DR) \\
\hline Qualifications & $\begin{array}{l}\text { Doctor of } \\
\text { Professional } \\
\text { Studies }\end{array}$ & PHD & MSc Cardiac Imaging & PHD \\
Profession & $\begin{array}{l}\text { Clinical Scientist } \\
\text { Relationship }\end{array}$ & Desearch Fellow & $\begin{array}{l}\text { Clinical Scientist and Scientific } \\
\text { Lead }\end{array}$ & Professor of Health Sciences \\
\hline
\end{tabular}

Although data saturation was achieved at around 20 patients, in line with similar studies the MV sampling criteria for gender and ethnicity was not sufficient. ${ }^{22} 46$ Therefore, recruitment continued until 30 patients were enrolled to improve minority variation in the study. Despite the continued recruitment, Black male participants remained unrepresented.

\section{Data collection}

Data were collected from February 2014 to October 2015. All interviews were conducted by NS, who received qualitative research training prior to data collection and analysis. The interviews were regularly discussed with the other authors DR and AC; author characteristics are detailed in table 3 .

The interview guide (online supplemental file 1) was initially informed by current literature on symptom interpretation and help-seeking for CHD, as well as by discussions with chest pain specialists, specifically cardiac scientists and nurses. Later, the guide was informed by the interview process itself. The guide was piloted with two participants and revisions were made to allow for greater scope for broader participant narratives. Throughout the interview process, the guide was refined by NS following discussion with the research team (DR and AC) to allow for the exploration of emerging areas.

For convenience, interviews were held on the same day as patients' RACPC appointments and were conducted in one of the cardiology department offices to avoid interruptions. Prior to the interviews commencing, introductions were made and key points from the participant information sheet were reiterated: participation was voluntary and non-participation would not have any bearing on care. An opportunity to ask any further questions was provided.

During the interview participants were asked broad questions, for example, 'Tell me about the symptoms that led you to see your GP'. They were encouraged to tell their own narrative and were not interrupted. During natural breaks in the patient narratives, probing was used to zone in on key issues, for example, 'How severe were your symptoms?' The interviews lasted approximately $45 \mathrm{~min}$, were audio recorded and transcribed verbatim. All digital recordings were checked by NS and labelled with a unique study number only for anonymity.

\section{Data analysis}

The analysis was conducted in parallel with data collection and comprised four phases: recording the interview observations; coding and coding queries; organising the themes; rerunning coding queries and rechecking themes. Observation notes were completed for each interview to record overall impressions. An initial paper-based line-by-line coding was undertaken after the first four interviews, and $48 \mathrm{draft}$ codes were then identified. Later, an additional 11 codes were added following further interviews. After discussions with the research team (NS, $\mathrm{AC}$ and DR) codes were streamlined by merging similar codes, which resulted in a working set of 44 codes (online supplemental file 2). In the second phase, transcripts and draft codes were entered into NVivo, and coding queries were used to explore relationships between different codes, for example, 'symptoms', 'severity' and 'helpseeking'. Data codes were also explored within sample dimensions, for example, 'women' and 'help-seeking'. Tabulation was used to begin the thematic analysis and to organise themes. Initially, four overarching themes were identified: 'chest pain and CHD symptoms'; 'helpseeking practices'; 'CHD risk and behaviours'; and 'test and treatments'. Following discussions with the research team, to better reflect the data, themes were reorganised into three main headings: 'symptoms'; 'barriers and enablers of help-seeking'; and 'seeking help from a GP'. For the fourth phase as an iterative process, NS reran the coding queries and compared them to the initial analysis for accuracy and consistency.

\section{Patient and public involvement}

There was no formal Patient and Public Involvement (PPI) in the design phase as it was not required at the time by the ethics committee. In addition, the study was of small scale, and carried out in a short time frame to fit National Health Service (NHS) capacity. However, PPI was undertaken in the dissemination of findings, where patients were involved in the development of a leaflet to improve CHD help-seeking and symptom interpretation. ${ }^{47}$

\section{FINDINGS}

Findings are reported around three key themes: 'making sense of symptoms'; 'barriers and enablers of helpseeking'; and 'seeking help from a GP'. Participant quotes are provided to support findings presented in themes; 
quotes are followed by sex, age, ethnicity, job area and when the interview was undertaken (pre/post receiving RACPC results) in order to provide context.

\section{Making sense of symptoms}

Attributing symptoms can be understood as a threestage process: first, if the participant believed that a new sensation in their body was a medical symptom; second, whether that symptom needed to be acted on; and third, if the participant believed that the symptom could have a potential cardiac cause. For a participant to assign a 'medical status' to a symptom it had to be new, unfamiliar, troublesome and persistent. It did not necessarily have to be painful, although it often was.

PA03. 'I wouldn't call it pain, no, I would more call it abnormal, un-normal.' (Male, 29, White, Management) (Pre)

PA19. 'Basically, I had a pain here that went a little bit down my arm, and it was a pain that I didn't recognise.' (Female, 77, Asian, Education) (Post)

Symptoms were not initially assigned a medical status; they were normalised or even dismissed, for example, as age-related pain or fitness-related, or attributed to preexisting conditions. Some participants, who were younger and otherwise healthy, believed their symptoms were psychosomatic. Here, participants reported thinking that their symptoms were partially imagined or exacerbated because of stress and worry about them, or believing that the body was 'releasing stress'. These factors all delayed seeking help.

PA17. 'If anything did slow me down it was that psychosomatic thing $[. .$.$] my worry if I was just imagin-$ ing it [... I I am quite youngish and it in all honesty it was not likely to be something too dramatic.' (Male, 46, White, Management) (Post)

PA11. 'I was worrying about having these symptoms and I did think that was probably part of it, the more worry about it the worse it gets. I don't know what you call it when you make symptoms worse by worrying it. Psychosomatic. But there was no denying that there was a physical pain.' (Male, 29, White, Media) (Post)

Even after a 'medical status' was assigned, symptoms were not necessarily acted on; in many cases, participants attempted a variety of self-management strategies. It was only when these strategies failed, and their symptoms continued or worsened, that further steps were likely to be taken.

PA09. 'I tried to drink less coffee for a bit but that's quite difficult, and I tried to focus a little bit more on my diet.' (Female, 27, White, Legal) (Pre)

For the medical symptoms to be attributed as having cardiac origins, other influences came into play. The extent to which participants believed they were a 'coronary candidate' (lay beliefs regarding who gets CHD), played an important part in symptom attribution. Participants used their own behaviours and lifestyle as reference points for considering the likelihood their symptoms were heart-related. Smoking, drug use, alcohol consumption and stress were among the perceived high-risk behaviours that concerned patients. Family history and past personal experience of heart disease also played a role.

PA04. 'I've had a number of stress-related issues in the last 2 years [...] I'm a young guy but I'm not very fit, I drink a lot $[\ldots]$. So that prompted me to connect more dots than were there, probably thinking it is cardiac' (Male, 37, White, Management) (Post)

PA08. 'I've had three or four heart attacks before $[\ldots]$. There's something wrong [...] I had previous stents fitted [...], I've felt a million dollars but since the last time I haven't.' (Male, 54, White, Business Owner) (Pre)

Some participants had pre-existing conditions that added further complexity to the attribution of their symptoms as they linked them to their underlying illnesses. It was only when their symptoms either did not resolve or worsened that they considered CHD as a possible cause.

PA09 'I first went (GP) about 2 years go (chest pain) and they said it was mastodynia. Then I started having spasms (in chest area) and nose bleeds at same time so I thought I better try and force it through a bit so I talked to my GP again.' (Female, 27, White, Legal) (Pre)

\section{Barriers and enablers of help-seeking}

Some participants were initially reluctant to speak to friends and family about their symptoms, so as not to burden them. When they did share, they were often selective; parents would avoid telling their children and some would avoid telling their spouses if they were the main caregiver or if their spouse was ill. Indeed, the need to care for an ill partner could act as an enabler for professional help-seeking.

PA23. 'Well I wanted to go to the doctor because of the dizziness. I wouldn't want him [my husband] to come back and find me on the floor because of his heart problems' (Female, 76, White, Administration) (Pre)

For other participants social isolation meant that they simply did not have access to friends or family to discuss symptoms. Often this isolation was more prevalent in ethnic minority groups because their family was overseas. One widowed participant of Arabic heritage with a traumatic childhood talked about how her personal experiences and cultural influences led to her being isolated.

PA05 'My mother died when I was 4years old. My father was Iraqi, he had a lot of wives and one day my mother was there and next day she disappeared. I am self-reliant. I don't like to say I have got this or 
that unless I am really really ill [...] I'm not very good with people. I'm slightly solitary' (Female, 76, Arabic, Retail) (Post)

However, being alone or living alone at the time of symptoms could also aid prompt professional help-seeking.

PA26. 'I have a symptom because I was alone, like I get pain and I can't breathe like that, you know what I did, I just ring the ambulance to go' (Female, 72, Asian, Artisan) (Post)

Other participants were able to talk about their symptoms with friends and family and it was often a first step towards formal help-seeking. In one case, a participant of Asian heritage talked about having multiple influencers-his partner, children and colleagues. They not only encouraged him to seek professional help, but also to make his employer aware he had health issues which may affect his work performance.

PA12. 'I told my wife that, oh, I am having this, I told my children basically. They just said go to the GP and tell my line manager as well that what I am feeling $[\ldots]$ they might think I'm lazy or something like that' (Male, 47, Asian, Civil Servant) (Pre)

Validation of symptoms by friends and family also prompted professional help-seeking, especially when they were health professionals. However, this was not always the case, some friends and family downplayed or dismissed symptoms, thereby hindering formal help-seeking.

PA20. 'And I have friends who are ex-nurses and they said, look just don't mess around with it, get it checked out'. (Female, 48, White, Artisan) (Pre)

PA03. 'They say, ah don't worry, it's nothing, you're not going to die.' (Male, 29, White, Management) (Pre)

Many participants were reluctant to seek professional help from GPs. They were cognisant of the pressures on doctors' time and the health system as a whole, so they took care not to 'waste their time' with 'trivial' health concerns. Other participants adopted strategies they imagined would save their doctor's time, including storing up symptoms in order to present a clear picture and avoid being dismissed by a busy doctor.

PA09. 'Doctors are under a lot of time constraint and a lot of pressures, and when one goes to the doctor one needs to have exactly the symptoms that one can gather together and put them all in front of them, otherwise it's usually going to be dismissed or you might sound like a hypochondriac.' (Female, 27, White, Legal) (Pre)

However, conversely, other participants had no doctor reluctance. In these cases, a positive relationship with their GP appeared to support help-seeking.

PA22. 'No, I do see my doctor [...] I've had for many, many years. So, it may sound awful, I actually quite enjoy seeing him.' (Male, 54, White, Business Owner) (Post)

Time and accessibility to GP services impacted professional help-seeking. Many participants highlighted complex booking processes and long appointment waiting times as issues, especially if they worked full-time or had other commitments. However, if participants' GPs were accessible, as they had pre-booked appointments or were consulting for other matters, they sought assistance for their potential CHD symptoms immediately.

PA12. 'It is the time, waiting time to see GP and particularly finding time as well, either you have to find time to book an appointment in the morning or late evening, then you have to change your work schedule as well.' (Male, 47, Asian, Civil Servant) (Post)

PA05. 'About three weeks ago, I had an appointment with the doctor (routine monitoring) and I walked fast and I got this pain in my chest pain. He said to me "Are you ok?" And I said, "yes, but I have got this funny pain in my chest." That was the start of all this about sending me to cardiology.' (Female, 76, Asian, Retail) (Post)

Popular media (specifically television) were linked to a reluctance to seek help for some participants. Participants talked about 'typical heart symptoms' they had seen in the media such as sweating, extreme pain, collapsing with a gripping pain in the chest. They were reluctant to seek help when their milder symptoms did not match this depiction. However, media health campaigns also encouraged help-seeking. One participant was prompted to seek help when they recalled a television (TV) campaign about CHD; another was prompted by a high-profile TV campaign about lung cancer.

SUBP01. 'My perception of a heart attack is someone flaking out on the floor $[\ldots]$ you see people, on TV, having heart attacks, and that wasn't me [...] I am not going to make a fool of myself by queuing up in A\&E.' (Female, 59, White, Administration) (Post)

PA 11. 'At the moment there is this NHS campaign about cough and lung cancer, it is everywhere at the moment $[\ldots]$ prompted me to go the doctor for this cough more so than the chest pain.' (Male, 29, White, Media) (Post)

Barriers to help-seeking contributed to a delay in consulting a professional. In some cases it was a few hours, but in others it was weeks or even months. Although variable, some of the shortest delays tended to be reported among White male participants in higher-income professional occupations, for example, business owners. Some of the longer delays tended to be reported by ethnic minorities.

PA22. 'You know, 12 hours later.' (Male, 54, White, Business Owner) (Post) 
PA13. 'It's about 6 months ago [...] I had a problem with breathing. And it's a little bit of pain on my left side, so it's chest pain.' (Female, 25, Asian, Artisan) (Post)

\section{Seeking help from a GP}

Once the decision to seek professional help was made, participants consulted their GPs. Presentations to GPs were complex, including a range of symptoms with varying degrees of severity and risk factors, as well as some pre-existing conditions. Some participants, who had clear typical cardiac symptoms with a relevant history and risk factors, were referred immediately to RACPC. In other cases, where symptoms were less typical, a referral was still made if it was evident that reassuring the patient was a helpful thing to do.

PA20. 'I went to my GP after having chest pains after about 2 weeks [...] I saw [my doctor] who got quite concerned and she said to me, given your history, your background, your parents, I'm going to refer to the chest pain clinic.' (Female, 48, White, Artisan) (Pre)

PA17. 'He [my doctor] said it probably was something muscular but nevertheless you're identifying it as maybe to do with your heart and this would be a useful thing for you to do. I was grateful that he did that.' (Male, 46, White, Senior Management) (Post)

Other participants with non-specific symptoms or preexisting conditions which might produce similar symptoms were not always referred immediately. Multiple visits were sometimes required before referral as their health concerns persisted, worsened and/or changed.

PA11. 'Around 6 months before this appointment [...] I started having a cough related to a chest pain, after a month, I thought I'd go to the doctors who said it was asthma. It came and went over a 6 month period [...] So I went to a GP [different one] a couple of weeks ago because for seven consecutive days, I had a pain in my chest pain and the GP referred to RACPC' (Male, 29, White, Media) (Post)

\section{DISCUSSION}

Among this novel research on RACPC patients we found that patients actively interpreted the importance of their symptoms with or without the help of significant others. Overall, there were similar challenges around interpreting symptoms and help-seeking practices in the RACPC context as reported in the ACS literature. We also found that several factors including coronary candidacy, access to medical services, family and friends could play a contradictory role in help-seeking and sometimes encouraged a delay. Media could also have conflicting influences on help-seeking.

As other health research has noted, the interpretation of symptoms involved several decisions and processes. ${ }^{16}{ }^{36} \mathrm{In}$ this study, participants considered whether a new symptom was important, what its cause was and whether it needed to be acted on. In general, new, unknown, bothersome and persistent physical sensations were classified as symptoms requiring medical help. They did not necessarily need to be painful or severe although they often were, which increased the likelihood of prompt help-seeking. In other studies, it has been noted that the severity of symptoms influences how quickly they are acted on. ${ }^{14} 1948$

Even after a potential medical status was assigned to symptoms, initial actions undertaken by many participants included dismissing or normalising symptoms as age-related, fitness-related or related to pre-existing health complaints. Others attempted to self-manage their symptoms. It was only after non-resolution and progression participants reappraised their symptoms and took steps to obtain lay and/or professional assistance. Similar responses have been described by studies on ACS and patients with cancer. ${ }^{16-19} 343749$ We also found that believing symptoms had a psychosomatic element was present among a few participants. Some believed themselves to be healthy thus their symptoms were unlikely to be significant; others believed their symptoms were exacerbated by worry. This was not found in ACS studies and may be a result of the milder symptoms experienced by this cohort of patients. For a potential cardiac cause to be assigned, our findings generally echoed the literature on patients in an ACS context. The type and locality of pain were important. ${ }^{19}$ Coronary candidacy was also evaluated based on lifestyle and family history, ${ }^{12}{ }^{14}$ and current symptoms were referenced against knowledge of CHD through personal experience or the experiences of others. $^{161822}$

The reluctance to seek professional help reported in ACS studies was also raised by our participants, including being mindful of the doctors' time and not wanting to be seen as wasting time with unimportant health concerns. ${ }^{1421}$ Participants also adopted strategies they imagined would save their doctor time, including collating symptoms to present a clear picture to the doctor or not asking about secondary health complaints. Other health research has noted that patients take active steps to save doctors' time, but they did not raise the specific strategies described in this study as highlighted above. ${ }^{50-52}$ Another factor, noted in this research, that could deter help-seeking was difficulty accessing medical services. This is echoed by other studies that found time-consuming, inconvenient or complex processes to access medical services could act as a barrier to help-seeking. ${ }^{14} 192753$ This was a particular issue for participants who had their own business, as a significant absence would have a direct financial impact. ${ }^{3054-56}$ However, we also found, as other works have done, that accessible GPs, for example, pre-book appointment or consulting for another matter, enabled help-seeking. ${ }^{36}$

Many factors influencing help-seeking identified by this study could also have a contradictory role. Friends and family, as other studies have noted, could validate symptoms and encourage help-seeking, ${ }^{1357}$ other times, they 
downplayed symptoms for reassurance, hindering professional help-seeking. ${ }^{14} 19$ The role of caregiver and financial provider was also contradictory in this study, as other works have found. ${ }^{12}{ }^{1357}$ In some cases, participants prioritised their care duties and work commitments above their own health needs. For others, the need to be healthy and to be able to provide care or financial stability acted as an enabler to get medical attention. Coronary candidacy among participants also had contrasting impacts on helpseeking, as other research has noted. ${ }^{12} 1416$ The more participants believed they were at risk through information gathered in the media, family history or lifestyle, the more promptly they acted. However, the converse was also true and could impede help-seeking.

Popular media and health campaigns also played a role in both symptom interpretation and help-seeking. Among our participants, as in other studies in an ACS context, media and public health campaigns can promote helpseeking by creating awareness of conditions and how to access help. ${ }^{21}{ }^{22}$ Interestingly, our study highlighted that these campaigns did not even need to be in the area of CHD. Campaigns for other conditions encouraging helpseeking for minor persistent symptoms linking them to potentially more serious issues seemed to give permission to seek medical attention more generally. However, there are now concerns that the COVID-19 'Stay home, save lives' campaign ${ }^{24}$ might be having unintended consequences on help-seeking, as accessing medical services for CHD and other non-COVID-19 illnesses has dropped significantly in the UK and the rest of Europe. ${ }^{25}{ }^{26}$ Additionally, as other authors have noted, participants could be reluctant to seek help if their symptoms were not as they had seen in the media. ${ }^{16} 1823$ These studies highlighted the effects of the 'Hollywood heart attack' with dramatic symptoms often portrayed on TV, discouraging help-seeking in those with milder symptoms.

In our study, as in studies based on ACS, many participants delayed seeking help. ${ }^{48}{ }^{29}$ We found that several factors influenced help-seeking delay including coronary candidacy, accessibility of medical services and friends and family, as highlighted above. Culture and family also intersected with these issues. Some ethnic minorities in our study were more removed from their overseas family and were unable to get support for their health issues. Other research has also found immigrants with cultural differences can become socially isolated causing negative health consequences. ${ }^{58}$ However, for others helpseeking was encouraged by their larger extended families supporting help-seeking. In addition, the need to be healthy to support family as an enabler to help-seeking was noted among some Asian men. Other works have also highlighted potential cultural differences between western and non-westernised approaches to help-seeking among men. ${ }^{57}$ Overall, while our study did observe cultural circumstances that influence help-seeking, more examination in this area is needed.

Referrals to the RACPC are via primary care which acts as the gateway to specialist services. In our cohort GPs needed to be convinced patients' symptoms justified a referral. It appeared GPs did not only consider typical or classical CHD symptoms when making a decision to refer, they were also sensitive to patients' emotional concerns and need for reassurance. While referrals for reassurance can be helpful for patients, they may have limited reach for those who are not able or willing to communicate this need to a GP. Indeed, a study in the field of medically unexplained symptoms-sometimes attributed to chest pain - noted patients are not always comfortable sharing the emotional aspects of their health concerns. ${ }^{59}$ Additionally, cultural differences and language barriers have been found to present communication challenges in primary care, acting as obstacles to diagnosis and treatment for patients. ${ }^{60}$ However, research into referral practices for CHD has highlighted GPs use non-explicit clues and non-verbal cues: in one Belgian study, GPs reported using observations including a tendency to downplay symptoms, changes in daily routine, physical appearance and their own 'gut feeling' about the patient when deciding to refer. ${ }^{61}$ The help-seeking experiences of patients in primary care in the context of accessing the RACPC pathway is an area requiring further exploration.

\section{Strengths and limitations}

Our study has several limitations. First, there was no PPI design phase of this study; future studies should include PPI in the design phase as it may improve data collection and interpretation of findings. Second, some participants had symptoms for a period of time before seeking help and their accounts may have been influenced by a degree of recall bias (table 2). Third, we only interviewed participants at RACPC at a single centre based in London. Fourth, despite over-enrolment, participants of Black ethnicity were under-represented in the study. Aside from this under-representation, we believe the strength of this study is the diverse range of experiences and insights obtained.

\section{CONCLUSION}

This study explored help-seeking experiences of patients in relation to interpreting their symptoms, deciding to seek help, seeking advice from family and friends and accessing medical services. Qualitative evidence is important to inform primary care and public health approaches. This study's findings suggest that interpreting symptoms is challenging for patients of RACPCs and this can contribute to a reluctance to seek professional help. This work also sheds light on GPs responses and referral practices for CHD-related symptoms. It revealed that GPs consider other factors in addition to symptom presentation when making referral decisions, such as the need for reassurance. However, this may be limiting where patients are not comfortable or unable to communicate their health concerns. Media and public campaigns, have the potential to be confusing, yet can have benefits in encouraging appropriate help-seeking. Insights gathered from 
this study were used to develop a patient leaflet for the NHS focussed on the importance of acting promptly for symptoms even if they are mild.

\section{Twitter Nolan Stain @drnolanstain}

Acknowledgements The University of Westminster for sponsoring this study, and Dr Patricia Maitland and Dr Bob Odle for their help in obtaining this sponsorship. Queen Mary's Roehampton's (Cardiology Department) for agreeing to be the host site for this study: with special gratitude to Lucy Titheridge and Mona Chauhan for their support and assistance. Dr Beth Unsworth for helping me conceptualise this study. Dr Bernadette Kirwan, my dyslexia tutor, for supporting me through this research process. Finally, The Late 'Lady' Henja Osgood for her continued input throughout this research process.

Contributors NS conceived the study with input and guidance from DR and AC. NS conducted the interviews. NS analysed the interviews with support from DR and AC NS drafted the first version of the manuscript. NS and CR revised and restructured the manuscript. DR and AC reviewed and provided input to all drafts. All authors critically read, revised and approved the final manuscript. NS is guarantor. NS is a clinical scientist and was a Professional Doctorate candidate at the time of conducting the study. DR is a Professor of Health Sciences and Research Director of the College of Liberal Arts and Sciences at the University of Westminster. AC is Research Fellow at the University of Westminster. CR is a clinical scientist and Scientific Lead at NHS England.

Funding The authors have not declared a specific grant for this research from any funding agency in the public, commercial or not-for-profit sectors.

Competing interests None declared.

Patient consent for publication Not required.

Ethics approval NHS Health Research Authority Fulham Research Committee granted approval (Reference no. 14/L0/0169). Research and Development approval was granted from St George's Joint Enterprise Research Office (Reference no. 14.0007)

Provenance and peer review Not commissioned; externally peer reviewed.

Data availability statement All data relevant to the study are included in the article or uploaded as supplementary information. Since complete transcripts of interviews potentially allow for identification of individuals, complete transcripts are not provided.

Supplemental material This content has been supplied by the author(s). It has not been vetted by BMJ Publishing Group Limited (BMJ) and may not have been peer-reviewed. Any opinions or recommendations discussed are solely those of the author(s) and are not endorsed by BMJ. BMJ disclaims all liability and responsibility arising from any reliance placed on the content. Where the content includes any translated material, BMJ does not warrant the accuracy and reliability of the translations (including but not limited to local regulations, clinical guidelines, terminology, drug names and drug dosages), and is not responsible for any error and/or omissions arising from translation and adaptation or otherwise.

Open access This is an open access article distributed in accordance with the Creative Commons Attribution Non Commercial (CC BY-NC 4.0) license, which permits others to distribute, remix, adapt, build upon this work non-commercially, and license their derivative works on different terms, provided the original work is properly cited, appropriate credit is given, any changes made indicated, and the use is non-commercial. See: http://creativecommons.org/licenses/by-nc/4.0/.

\section{ORCID iD}

Nolan Stain http://orcid.org/0000-0002-5813-6862

\section{REFERENCES}

1 Timmis A, Townsend N, Gale CP, et al. European Society of cardiology: cardiovascular disease statistics 2019. Eur Heart J 2020;41:12-85

2 Haasenritter J, Stanze D, Widera G, et al. Does the patient with chest pain have a coronary heart disease? Diagnostic value of single symptoms and signs--a meta-analysis. Croat Med J 2012;53:432-41.

3 Daly C, Clemens F, Lopez Sendon JL, et al. Gender differences in the management and clinical outcome of stable angina. Circulation 2006;113:490-8.
4 Meyer MR, Bernheim AM, Kurz DJ, et al. Gender differences in patient and system delay for primary percutaneous coronary intervention: current trends in a Swiss ST-segment elevation myocardial infarction population. Eur Heart J Acute Cardiovasc Care 2019;8:283-90.

5 NICE. Clincal Knowlegde summaries: chest pain online NICE 2015. Available: http://cks.nice.org.uk/chest-pain\#!diagnosissub:8 [Accessed April 2015].

6 Department of Health. National service framework (NSF) for coronary heart disease (CHD). London HMSO, 2000.

7 Mathieson S, Victor K, Jarrett-Smith L, et al. The rapid access chest pain clinic (RACPC) and the role of the cardiac physiologist. British Journal of Cardiac Nursing 2017;12:300-5.

8 Stain N, Ross C, Leitch M, et al. The development of allied professions-led cardiac clinics. ACNAP Essentials 4 You. European Journal of Cardiovascular Nursing 2020;19:S1-68.

9 Boyle RM. Value of rapid-access chest pain clinics. Heart 2007;93:415-6.

10 Cornally N, McCarthy G. Help-Seeking behaviour: a concept analysis. Int J Nurs Pract 2011;17:280-8.

11 Rickwood D, Deane FP, Wilson C, et al. Young people's help-seeking for mental health problems. Advances in Mental Health 2005;4.

12 Lockyer L. Women's interpretation of their coronary heart disease symptoms. Eur J Cardiovasc Nurs 2005;4:29-35.

13 Farrimond $\mathrm{H}$. Beyond the caveman: rethinking masculinity in relation to men's help-seeking. Health 2012;16:208-25.

14 Emslie C. Women, men and coronary heart disease: a review of the qualitative literature. J Adv Nurs 2005;51:382-95.

15 Galdas PM, Johnson JL, Percy ME, et al. Help seeking for cardiac symptoms: beyond the masculine-feminine binary. Soc Sci Med 2010;71:18-24.

16 Baxter SK, Allmark P. Reducing the time-lag between onset of chest pain and seeking professional medical help: a theory-based review. BMC Med Res Methodol 2013;13:1.

17 Moser DK, McKinley S, Dracup K, et al. Gender differences in reasons patients delay in seeking treatment for acute myocardial infarction symptoms. Patient Educ Couns 2005;56:45-54.

18 Zerwic JJ, Ryan CJ, DeVon HA, et al. Treatment seeking for acute myocardial infarction symptoms: differences in delay across sex and race. Nurs Res 2003;52:159-67.

19 Gyberg A, Björck L, Nielsen S, et al. Women's help-seeking behaviour during a first acute myocardial infarction. Scand J Caring Sci 2016;30:670-7.

20 Kirchberger I, Heier M, Wende R, et al. The patient's interpretation of myocardial infarction symptoms and its role in the decision process to seek treatment: the MONICA/KORA myocardial infarction registry. Clin Res Cardiol 2012;101:909-16.

21 Turris SA, Finamore S. Reducing delay for women seeking treatment in the emergency department for symptoms of potential cardiac illness. J Emerg Nurs 2008;34:509-15.

22 Foster S, Mallik M. A comparative study of differences in the referral behaviour patterns of men and women who have experienced cardiac-related chest pain. Intensive Crit Care Nurs 1998;14:192-202.

23 Tullmann DF, Dracup K. Knowledge of heart attack symptoms in older men and women at risk for acute myocardial infarction. $J$ Cardiopulm Rehabil 2005;25:33-9.

24 England PH. Stay at home: guidance for households with possible coronavirus (COVID-19) infection. In: Do H, ed. London, 2020.

25 Metzler B, Siostrzonek P, Binder RK, et al. Decline of acute coronary syndrome admissions in Austria since the outbreak of COVID-19: the pandemic response causes cardiac collateral damage. Eur Heart $J$ 2020;41:1852-3.

26 England PH. Emergency department: syndromic surveillance system. In: England PH, ed. London: UK: Government Asset Publishing, 2020.

27 Tod AM, Read C, Lacey A, et al. Barriers to uptake of services for coronary heart disease: qualitative study. BMJ 2001;323:214-7.

28 Adamson J, Donovan J, Ben-Shlomo Y, et al. Age and sex interaction in reported help seeking in response to chest pain. $\mathrm{Br} J$ Gen Pract 2008;58:318-23.

29 Pastorius Benziger C, Bernabe-Ortiz A, Miranda JJ, et al. Sex differences in health care-seeking behavior for acute coronary syndrome in a low income country, Peru. Crit Pathw Cardiol 2011;10:99-103.

30 Noureddine S, Arevian M, Adra M, et al. Response to signs and symptoms of acute coronary syndrome: differences between Lebanese men and women. Am J Crit Care 2008;17:26-35.

31 van Tiel D, van Vliet KP, Moerman CJ. Sex differences in illness beliefs and illness behaviour in patients with suspected coronary artery disease. Patient Educ Couns 1998;33:143-7. 
32 Maas A, van der Schouw YT, Regitz-Zagrosek V, et al. Red alert for women's heart: the urgent need for more research and knowledge on cardiovascular disease in women, 2011: 1362-8.

33 Westerman S, Wenger NK, Wenger Nanette K. Women and heart disease, the underrecognized burden: sex differences, biases, and unmet clinical and research challenges. Clin Sci 2016;130:551-63.

34 Albarran JW, Clarke BA, Crawford J. 'It was not chest pain really, I can't explain it!' An exploratory study on the nature of symptoms experienced by women during their myocardial infarction. J Clin Nurs 2007;16:1292-301.

35 Higginson R. Women's help-seeking behaviour at the onset of myocardial infarction. Br J Nurs 2008;17:10-14.

36 Scott SE, Walter FM, Webster A, et al. The model of pathways to treatment: conceptualization and integration with existing theory. $\mathrm{Br} \mathrm{J}$ Health Psychol 2013;18:45-65.

37 Mills K, Birt L, Emery JD, et al. Understanding symptom appraisal and help-seeking in people with symptoms suggestive of pancreatic cancer: a qualitative study. BMJ Open 2017;7:e015682.

38 Birt L, Hall N, Emery J, et al. Responding to symptoms suggestive of lung cancer: a qualitative interview study. BMJ Open Respir Res 2014;1:e000067.

39 Hall N, Birt L, Banks J, et al. Symptom appraisal and healthcareseeking for symptoms suggestive of colorectal cancer: a qualitative study. BMJ Open 2015;5:e008448.

40 Elliot RT L. Descriptive and interpretive approaches to qualitative research. In: Miles JGP, ed. A Handbook of research methods for clinical and health psychology. Oxford, England: Oxford University Presss, 2005: 147-59.

41 Draper A, Swift JA. Qualitative research in nutrition and dietetics: data collection issues. J Hum Nutr Diet 2011;24:3-12.

42 Patton M. Qualitative evaluation and research methods. Beverley Hills, CA: Sage, 2002.

43 Stain N, Ridge D, Cheshire A. Gender comparisons in nonacute cardiac symptom recognition and subsequent helpseeking decisions: a mixed methods study protocol. BMJ Open 2014; 4:e005742.

44 O'Brien BC, Harris IB, Beckman TJ, et al. Standards for reporting qualitative research: a synthesis of recommendations. Acad Med 2014;89:1245-51.

45 Mirfin-Veitch B, Bray A, Ross N. "It was the hardest and most painful decision of my life!": seeking permanent out-of-home placement for sons and daughters with intellectual disabilities. J Intellect Dev Disabil 2003;28:99-111.

46 Baker SER. How many qualitative interviews is a enough, 2008.

47 Stain N. An exploration of the help-seeking experiences of men and women referred to a Rapid Access Chest Pain Clinic: a cardiac physiologist-managed clinic [Doctoral]. EThOS: University of Westminster, 2017.

48 Nguyen HL, Saczynski JS, Gore JM, et al. Age and sex differences in duration of prehospital delay in patients with acute myocardial infarction: a systematic review. Circ Cardiovasc Qual Outcomes 2010;3:82-92.

49 Bennett SJ, Cordes DK, Westmoreland G, et al. Self-Care strategies for symptom management in patients with chronic heart failure. Nurs Res 2000;49:139-45.

50 Robb K, Stubbings S, Ramirez A, et al. Public awareness of cancer in Britain: a population-based survey of adults. Br J Cancer 2009;101 Suppl 2:S18-23.

51 Pollock K, Mechanic D, Grime J. Patients' perceptions of entitlement to time in general practice consultations for depression: qualitative study * Commentary: Managing time appropriately in primary care. BMJ 2002;325:687.

52 Cromarty I. What do patients think about during their consultations? A qualitative study. Br J Gen Pract 1996;46:525-8.

53 Schoenberg NE, Peters JC, Drew EM. Unraveling the mysteries of timing: women's perceptions about time to treatment for cardiac symptoms. Soc Sci Med 2003;56:271-84.

54 Emslie C, Men HK. Masculinities and heart disease: a systematic review of the qualitative literature. Current Sociology 2009;57:155-91.

55 O'Brien R, Hart GJ, Hunt K. "Standing out from the herd": Men renegotiating masculinity in relation to their experience of illness. International Journal of Men's Health 2007;6.

56 Courtenay WH. Constructions of masculinity and their influence on men's well-being: a theory of gender and health. Soc Sci Med 2000;50:1385-401.

57 Galdas P, Cheater F, Marshall P. What is the role of masculinity in white and South Asian men's decisions to seek medical help for cardiac chest pain? J Health Serv Res Policy 2007;12:223-9.

58 Ajrouch KJ. Social isolation and loneliness among Arab American elders: cultural, social, and personal factors. Res Hum Dev 2008;5:44-59.

59 Peters S, Rogers A, Salmon P, et al. What do patients choose to tell their doctors? qualitative analysis of potential barriers to reattributing medically unexplained symptoms. J Gen Intern Med 2009;24:443-9.

60 Harris SM, Binder P-E, Sandal GM. General practitioners' experiences of clinical consultations with refugees suffering from mental health problems. Front Psychol 2020;11.

61 Bruyninckx R, Bruel AVden, Hannes K, et al. GPs' reasons for referral of patients with chest pain: a qualitative study. BMC Fam Pract 2009;10:55 